Journal of Advanced Research in Fluid Mechanics and Thermal Sciences

Journal homepage: www.akademiabaru.com/arfmts.html ISSN: 2289-7879

\title{
A Stress Mapping Immersed Boundary Method for Viscous Flows
}

\author{
Karim M. Ali ${ }^{1,}{ }^{*}$, Mohamed Madbouli ${ }^{1}$, Hany M. Hamouda ${ }^{2}$, Amr Guaily ${ }^{3,4}$ \\ Department of Aerospace Engineering, Faculty of Engineering, Cairo University, 12613 Giza, Egypt \\ Department of Mathematics, Deanship of Educational Services, Qassim University, P.O. Box 6595 Buraydah 51452, Saudi Arabia \\ Department of Engineering Mathematics and Physics, Faculty of Engineering, Cairo University, 12613 Giza, Egypt \\ Smart Engineering Systems Research Center (SESC), Nile University, 12588, Shaikh Zayed City, Egypt
}

ARTICLE INFO ABSTRACT

\section{Article history:}

Received 4 April 2021

Received in revised form 3 August 2021

Accepted 6 August 2021

Available online 28 September 2021

Keywords:

CFD; finite element; immersed boundary; interface; mapping

\begin{abstract}
This work introduces an immersed boundary method for two-dimensional simulation of incompressible Navier-Stokes equations. The method uses flow field mapping on the immersed boundary and performs a contour integration to calculate immersed boundary forces. This takes into account the relative location of the immersed boundary inside the background grid elements by using inverse distance weights, and also considers the curvature of the immersed boundary edges. The governing equations of the fluid mechanics are solved using a Galerkin-Least squares finite element formulation. The model is validated against a stationary and a vertically oscillating circular cylinder in a cross flow. The results of the model show acceptable accuracy when compared to experimental and numerical results.
\end{abstract}

\section{Introduction}

Advancements in the computational capabilities of computers has reflected on the complexity of the problems tackled by computational fluid dynamics (CFD). Using conventional body-fitted grids [15] has limited the ability to simulate problems with complex motions. Some of these techniques require re-gridding at some time during the simulation which adds a computational cost and provokes artificial numerical diffusion. However, immersed boundary techniques relaxed the restriction imposed by body-fitted grid methods by opening the gate for simulating multi-body problems with highly complex motions.

The literature of immersed boundary methods is full of various approaches. Jafari [6] presented a review of various immersed boundary methods which include, but not limited to, the marker and cell method developed by Harlow and Welsh [7], the volume of fluid method presented by Hirt [8], the level set method presented by Osher and Fedkiw [9], and also the work of Udaykumar and Mittal [10] and Francois and Shyy [11].

Generally, immersed boundary methods can be divided into interface capturing and interface tracking methods. In interface tracking, the interface location between different phases, different

\footnotetext{
* Corresponding author.

E-mail address: karim2020@cu.edu.eg
}

https://doi.org/10.37934/arfmts.87.3.120 
fluids or fluids and non-fluids is known either from an equations of motion model or a prescribed path. On the other hand, in interface capturing, instead of previous knowledge, the interface location is solved for like Bagabir et al., [12] did for capturing the interface in a compressible gas-gas flow and Duo-xing and De-liang [13] did to capture a fluid-fluid interface in a porous medium. Another classification for immersed boundary methods is finite/sharp interface treatment.

Some methods depend on modeling the interface between different physical regions by a finite thickness interface across which physical quantities are smoothed between its two end values. On the contrary, sharp interface techniques impose an abrupt change of physical quantities across the interface.

Immersed boundary methods introduce a mass conservation issue near the immersed boundary [14]. Various approaches are found in the literature of immersed boundary methods for treating this issue. In a continuous forcing approach, source terms are added to the governing equations, and have effect only near the interface [15-18]. Goldstein et al., [19] introduced another forcing term for the aim of having a velocity feedback control. The method was used for modeling startup flows over a circular cylinder at low Reynolds numbers. Angot el al. [20] presented the idea of a porous medium whose permeability is infinite for fluid regions, zero for solid regions and smoothed in between. Analogous to the continuous forcing approach is the discrete one. Generally, in the discrete forcing approach the forcing terms are added to the discretized form of the governing equations. The indirect boundary condition imposition method was introduced by Mohd-Yosuf [21] in which the forcing terms were extracted directly from the discretized form of the Navier-Stokes equations. A ghost cell can be defined as the cell through which the interface passes.

In the ghost cell finite difference approach, a generic flow variable is interpolated inside the ghost cell from the values of the surrounding cells [22]. The cut-cell finite volume method was introduced by Clarke [23]. In this method, the cell through which the interface passes is re-constructed to keep its fluid part only. Despite its higher accuracy, this method is limited due to the complexity of the reconstructed polyhdera in case of three dimensions and due to the additional work needed to reform the connectivity of the grid at each time step. Salih et al., [24] used a cut cell method mixed with local adaptive mesh refinement around the immersed boundary. The method was used in a fluid structure interaction simulation of thin shaped bodies.

In this work, instead of integrating flow fields on the background grid nodes, they are integrated along the contour of the immersed boundary itself. Figure 1 summarizes this methodology. Figure 1 (a) shows a general flow field smoothed between the solid and fluid regions. This exemplary field shows the stair-step region present at the interface between the solid and the fluid regions. It is customary that the fluid stresses are integrated over this region to compute the immersed boundary forces. The definition of this interface region is based on a location criterion to the edges of the immersed boundary, hence resulting in this stair-step form. To dismiss this situation the following is introduced. Figure 1(b) shows a segment of the immersed boundary represented by the dashed curve. For each vertex of the immersed boundary, the grid element containing this vertex maps the stress tensor of its fluid nodes only onto the immersed boundary vertex using an inverse distance weight. In this manner, the immersed boundary vertices are represented as virtual grid nodes whose flow field values are interpolated from the values of the actual grid nodes. Finally, Figure 1(c) shows an exemplary stress tensor magnitude (namely $\sqrt{\sigma: \sigma}$ with $\sigma$ being the Cauchy stress tensor) after the mapping process. The mapped stress distribution can be easily integrated over the edges of immersed boundary with any numerical integration method. By this, the relative position of the immersed boundary vertex inside a comprising grid element is taken into account in the inverse distance weight calculation. Also, the curvature of an immersed boundary can be easily accounted for in the integration process. 


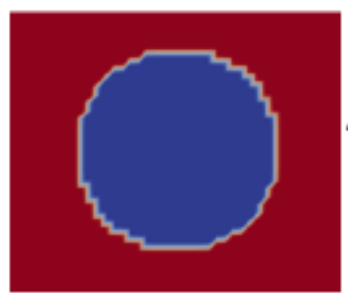

(a)



Fig. 1. (a) A general field smoothed between a solid (blue) region and a fluid (red) region. (b) A grid cell comprising a vertex with the fluid nodes $(F)$ mapping their stresses to the vertex. (c) The distribution of the mapped stresses magnitude along the contour of the immersed boundary

In this work, the pressure-stabilized incompressible Navier-Stokes equations $[25,26]$ were solved using a Galerkin-Least Squares (GLS) finite element method following [27]. Differentiating the solid and fluid regions was done by solving the point inclusion in a polygon using the dual perspective algorithm [28]. Solid regions were modeled as infinitely viscous fluid regions where the momentum equation simplifies to a rigid body motion condition. So, all the solid nodes have imposed velocities equal to that of the moving body. The continuity equation is solved for among the fluid as well as the solid regions to ensure a mass conservation inside the whole domain. Solving the continuity equation inside the solid regions with their velocities set to that of the body itself ensures that the pressure propagates correctly to the fluid regions near the cylinder. Such a technique enables the usage of Euler explicit forward time discretization, as it ensures that all the domain nodes possess history regarding their pressure and velocity. After presenting the methodology the model is validated against problems with stationary and moving bodies.

\section{Methodology}

\subsection{Governing Equations}

Brezzi and Pitk"aranta [25] introduced the pressure stabilization form of the Stokes equation. A generalization of this method to the unsteady Navier-Stokes equations takes the form

$$
\begin{aligned}
& \frac{\partial u}{\partial x}+\frac{\partial v}{\partial y}-\epsilon\left(\frac{\partial^{2} p}{\partial x^{2}}+\frac{\partial^{2} p}{\partial y^{2}}\right)=0 \\
& \frac{\partial u}{\partial t}+u \frac{\partial u}{\partial x}+v \frac{\partial u}{\partial y}+\frac{\partial p}{\partial x}-\frac{1}{R e}\left(\frac{\partial^{2} u}{\partial x^{2}}+\frac{\partial^{2} u}{\partial y^{2}}\right)=0,
\end{aligned}
$$


$\frac{\partial v}{\partial t}+u \frac{\partial v}{\partial x}+v \frac{\partial v}{\partial y}+\frac{\partial p}{\partial y}-\frac{1}{R e}\left(\frac{\partial^{2} v}{\partial x^{2}}+\frac{\partial^{2} v}{\partial y^{2}}\right)=0$

where $u$ and $v$ are the fluid's velocity components, $p$ is the fluid's pressure, and $\epsilon$ is a pressure stabilization parameter that is five to ten times the solution time step. Eq. (1)-(3) become the pressure stabilization form of the Stokes equation when $\epsilon$ is set to $\Delta t$ [29]. The Reynolds number $R e$ is defined as $\rho U_{\infty} L / \mu$ with $\rho$ being the fluid's density, $U_{\infty}$ being the characteristic flow velocity, $L$ being the characteristic length, and $\mu$ being the fluid's viscosity coefficient. The continuous flow field is discretized by the shape functions $\phi$ such that a continuous variable $g$ can be written inside a finite element as

$g \approx \tilde{g}=g_{\gamma} \phi_{\gamma}$

with the implicit summation of $\gamma$ being made over all the nodes of the finite element such that $g_{\gamma}$ represents the value of the approximate variable $\tilde{g}$ at the node $\gamma$. The superscript $(\sim)$ indicates an approximated variable. After discretization, the right hand sides of Eq. (1), (2) and (3) cease to vanish. These right hand sides become the non-zero residuals $R_{c}, R_{x}$ and $R_{y}$ for the continuity, $x$ direction momentum and $y$-direction momentum equations, respectively. These residuals are minimized by the weighting function $W$, such that for a computational domain $\Omega$, the volume integrals

$\int_{\Omega} R_{c} W d V=\int_{\Omega} R_{x} W d V=\int_{\Omega} R_{y} W d V=0$,

are satisfied. The superscript $(\sim)$ will be dropped from now on while taking into consideration that all subsequent flow variables are approximate variables stored in the grid nodes. To convert Eq. (5) to the weak form, second order derivatives are integrated by parts while omitting the resulting contour integrals around the domain boundary $\partial \Omega$ as they are overridden by Dirichlet boundary conditions or they naturally satisfy a zero Neumann boundary condition.

$W_{i}=\phi_{i}+\tau Q\left(\phi_{i}\right)$

$Q=\bar{u} \frac{\partial}{\partial x}+\bar{v} \frac{\partial}{\partial y}$

For a GLS formulation, the weighting function $W$ is a linear combination of the shape function $\phi$ and the differential operator $Q(\phi)$ for the differential equation at hand [27]. In Eq. (7), the definition of the differential operator $Q$ is stated with $\bar{u}$ and $\bar{v}$ being convection velocities that are approximated by the finite element averaged velocities from a previous time step. For a node $i$, the linear combination is made using a weighting parameter $\tau$ according to Eq. (6) with $\tau$ being defined as

$\tau=\left[\left(\frac{2}{\Delta t}\right)^{2}+\left(\frac{2 u_{e}}{L_{e}}\right)^{2}+\left(\frac{4}{\operatorname{Re} L_{e}^{2}}\right)^{2}\right]^{-0.5}$

In Eq. (8), $\Delta t$ is the solution time step, $u_{e}$ is an element averaged velocity, and $L_{e}$ is an element length scale. Only for the continuity equation, a standard Galerkin procedure is followed by setting $\tau$ to zero. Introducing Eq. (4) to Eq. (1)-(3) along with Eq. (5)-(8), and using Euler forward time 
discretization for the temporal derivatives results in a set of linear systems of equations. Using an index notation for compactness, these equations can be expressed as

$$
\begin{aligned}
& A_{\gamma \beta} p_{\beta}^{n}=-\frac{1}{\epsilon}\left(B_{\gamma \beta} u_{\beta}^{n}+C_{\gamma \beta} v_{\beta}^{n}\right), \\
& M_{\gamma \beta} u_{\beta}^{n+1}=\left(M_{\gamma \beta}-\Delta t K_{\gamma \beta}\right) u_{\beta}^{n}-\Delta t B_{\gamma \beta} p_{\beta}^{n}, \\
& M_{\gamma \beta} v_{\beta}^{n+1}=\left(M_{\gamma \beta}-\Delta t K_{\gamma \beta}\right) v_{\beta}^{n}-\Delta t C_{\gamma \beta} p_{\beta}^{n},
\end{aligned}
$$

In Eq. (9-11), the superscript $n$ refers to the time level. Eq. (9) is used to update the pressure field using the velocity field obtained from Eq. (10) and (11). The matrices $A, B, C, M$ and $K$ are defined for a finite element $\Omega_{e}$ with a volume of $V_{e}$ as follows

$$
\begin{aligned}
& A_{\gamma \beta}=\int_{\Omega_{e}} \frac{\partial \phi_{\gamma}}{\partial x_{k}} \frac{\partial \phi_{\beta}}{\partial x_{k}} d V_{e} . \\
& B_{\gamma \beta}=\int_{\Omega_{e}} \phi_{\gamma} \frac{\partial \phi_{\beta}}{\partial x} d V_{e}, C_{\gamma \beta}=\int_{\Omega_{e}} \phi_{\gamma} \frac{\partial \phi_{\beta}}{\partial y} d V_{e} . \\
& M_{\gamma \beta}=\int_{\Omega_{e}} \phi_{\gamma} \phi_{\beta} d V_{e} . \\
& K_{\gamma \beta}=\bar{u}_{i} \int_{\Omega_{e}} \phi_{\gamma} \frac{\partial \phi_{\beta}}{\partial x_{i}} d V_{e}+\tau \bar{u}_{k} \bar{u}_{l} \int_{\Omega_{e}} \frac{\partial \phi_{\gamma}}{\partial x_{k}} \frac{\partial \phi_{\beta}}{\partial x_{l}} d V_{e}+\frac{1}{R e} \int_{\Omega_{e}} \frac{\partial \phi_{\gamma}}{\partial x_{j}} \frac{\partial \phi_{\beta}}{\partial x_{j}} d V_{e} .
\end{aligned}
$$

In Eq. (12)-(15), the free indices $\gamma$ and $\beta$ correspond to the node order in the local finite element, while the dummy indices $i, j, k$ and $l$ correspond to variable order. For example, in Eq. (12), setting $k$ to one indicates a derivative with respect to the $x$ coordinate, while setting it to two indicates a

derivative with respect to the $y$ coordinate. The same analogy applies to Eq. (15) where $\bar{u}_{k}$ refers to $\bar{u}$ as well as $\bar{v}$ just by setting the index $k$ to one or two, respectively. The integrals in Eq. (12)-(14) depend on the grid shape only without any dependency on the flow variables, and hence are computed once at the beginning of the simulation and are stored to minimize the CPU time. The used finite elements local coordinates range from -1 to 1 on the computational axes $\zeta$ and $\eta$. Also, the shape function $\phi_{i}$ of the node $i$ in a local finite element is defined as

$\phi_{i}=\frac{1}{4}\left(1+\zeta_{i} \zeta\right)\left(1+\eta_{i} \eta\right)$

with $i$ ranging from one to four (the number of nodes of a quadrilateral).

\subsection{Stress Calculation Along the Moving Body}

The calculation of the forces acting on a moving body is very crucial for most applications. In traditional immersed boundary techniques, the forces acting on the immersed boundary are calculated by integrating the stresses over some of the background grid nodes. These nodes are mainly selected based on a location criterion relative to the immersed boundary edges. The locus of these nodes can result in a poor surface of integration, hence affecting the accuracy. To avoid this issue, the stress integration is made along the edges of the immersed boundary itself. This is done by 
mapping the flow field onto the vertices of the immersed boundary. The Cauchy stress tensor $\sigma$ of a fluid is defined using an index notation as

$\sigma_{\gamma \beta}=-p \delta_{\gamma \beta}+\frac{1}{R e}\left(\frac{\partial u_{\gamma}}{\partial x_{\beta}}+\frac{\partial u_{\beta}}{\partial x_{\gamma}}\right)$

where $\delta_{\gamma \beta}$ is the Kronecker delta function. Eq. (17) is written for a continuous domain. Re- writing the equation in a finite element context using an index notation and a standard Galerkin procedure results in

$$
\left(\sigma_{\gamma \beta}\right)_{j} \int_{\Omega_{e}} \phi_{i} \phi_{j} d V_{e}=-p_{k} \delta_{\gamma \beta} \int_{\Omega_{e}} \phi_{i} \phi_{k} d V_{e}+\frac{1}{R e}\left[\left(u_{\gamma}\right)_{s} \int_{\Omega_{e}} \phi_{i} \frac{\partial \phi_{s}}{\partial x_{\beta}} d V_{e}+\left(u_{\beta}\right)_{l} \int_{\Omega_{e}} \phi_{i} \frac{\partial \phi_{l}}{\partial x_{\gamma}} d V_{e}\right]
$$

where $\left(\sigma_{\gamma \beta}\right)_{j}$ is the stress tensor $\sigma_{\gamma \beta}$ at the node $j$, and $\left(u_{\gamma}\right)_{s}$ is the $\gamma$ component of the velocity field for the node $s$. For each of the vertices defining the immersed boundary, the grid element enclosing this vertex is defined. This grid element should by definition possess at least one grid node that is classified as inside the body by the dual perspective algorithm [28]. The Cauchy stress tensor at a body vertex inside this grid element can be obtained by interpolating the stress tensor of the element's fluid nodes using an inverse distance interpolation [30]. For an enclosing element having $N_{e}{ }^{\prime}$ fluid nodes, the Cauchy stress tensor at a vertex $i$ that is $d_{j}$ away from the fluid node $j$ is

$$
\left(\sigma_{\gamma \beta}\right)_{i}=\frac{\sum_{j=1}^{N_{e}^{\prime}} d_{j}^{-\kappa}\left(\sigma_{\gamma \beta}\right)_{j}}{\sum_{j=1}^{N_{e}^{\prime}} d_{j}^{-\kappa}}
$$

where, $\kappa$ is a power parameter. The mapped stresses are then integrated along the immersed boundary contour to get the resultant acting forces.

\subsection{Computational Procedure}

In the previous two sections, the equations and the mapping technique were stated. In this subsection, the procedure of the model is stated. One of the advantages of the presented model is that the background grid is time invariant in all its aspects. Hence, all grid related processes can be made only once at the beginning of the simulation and are stored for later usage. This behaviour may harm memory usage but is definitely in favour of CPU time consumption. Generally, scientific computations face a trade-off between memory usage and computation time. It is worth mentioning that the memory usage of the presented model is around 4.5 Gigabytes for a grid with a million nodes. This number may be different from one program to another. So, favouring the CPU time was not very harmful from a memory usage point of view.

The integrals of the shape functions $\phi$ are computed for each grid element using a GaussLegendre quadrature. Global matrices like the ones stated in Eq. (12-15) are highly sparse and are stored in a matrix compressed format. The locations of the non-zero cells in the global matrix depends on the connectivity of the grid's elements which is time invariant. So, for an exemplary node, identifying the global ID of the nodes that share a grid element with this node defines the location of the non-zero cells in the row corresponding to the exemplary node in a global matrix. 
The matrices of Eq. (12)-(14) are time invariant, while the matrix $K$ of Eq. (15) is dependent on the convection velocity. However, the element's volume integrals in Eq. (15) are time invariant. So, they can be computed and stored for each grid element to be used in filling the predefined nonzero locations in matrix $K$ at each time step.

Now, for each solution time step, the nodes of the grid are classified into fluid and solid nodes by using the dual perspective algorithm [28]. Moreover, when a simulated body is not moving, the nodal classification process regarding this body is done only once at the first time step. It is beneficial to define a virtual time invariant bounding box that encloses all the bodies during all the times. The purpose of this virtual bounding box is to make an initial nodal classification before the time iterations begin to determine the nodes inside the bounding box. Nodes that are outside this bounding box are always fluid nodes and hence are eliminated from any nodal classification process. Nodes that are classified as solid nodes are given a Dirichlet velocity boundary condition according to the velocity of their enclosing body.

Moreover, the weighting parameter $\tau$ is calculated for each element according to Eq. (8), and then, along with the velocities of the previous time step, is used to calculate the matrix $K$ according to Eq. (15). Eq. (9) is used to update the pressure field by first forming its right hand side and imposing pressure boundary conditions. Eq. (10) and (11) are solved for the new velocity field after imposing the Dirichlet boundary conditions of the solid nodes along with the wall nodes, velocity inlet nodes, and symmetry planes nodes. After obtaining the pressure and velocity fields, the Cauchy stress tensor can be calculated at grid nodes that are relevant to the mapping process according to Eq. (18). Note that the shape functions integrals in Eq. (18) are time invariant and are among the integrals calculated before the time iterations begin. To map the Cauchy stress tensor on the vertices of the moving body, the grid element enclosing a moving body vertex is searched for. To speed up this process, the closest grid node to each moving body vertex is determined during the nodes classification process. The grid element enclosing the moving body vertex is one of the grid elements sharing its closest grid node. After identifying the enclosing grid element, Eq. (19) is used to map the stresses of the fluid nodes of the enclosing grid element onto the moving body vertex. These mapped stresses are then integrated along the contour of the moving body using the edges' normal vectors. Such a process is repeated for every moving body in the domain.

\section{Results}

The flow over a circular cylinder whether oscillating or stationary is quite important for studying off-shore structures as well as heat exchangers that are subjected to induced vibrations. Experimental work for the oscillating circular cylinder can be found in [31-33]. Numerical simulations are also present like that discussed in [34-42].

\subsection{Flow Over a Stationary Circular Cylinder}

Persillon and Braza [43] discussed the effect of the domain size on the accuracy of CFD simulations of circular cylinders especially in small Reynolds number simulations. According to their conclusions, the rectangular domain is constructed to have the dimensions shown in Figure 2.

The cylinder is placed in a virtual bounding box with square grid elements of length $\delta_{e}$ referenced to the cylinder diameter. To select a proper value for $\delta_{e}$, a grid convergence study is conducted by varying the value of $\delta_{e}$ and measuring the natural frequency $f_{o}$ of the generated lift signal for a stationary circular cylinder at a Reynolds number of 185 . The value of $\delta_{e}$ is reduced until the natural shedding frequency stabilizes whilst keeping $\delta_{e}$ at acceptable levels for computational practicality. 
Figure 3 shows the grid convergence analysis for the selection of $\delta_{e}$. The figure shows that at a value of 0.01 for $\delta_{e}$, the changes in the natural shedding frequency is almost insignificant. Reducing $\delta_{e}$ below 0.01 would raise the size of the grid with no significant gains. Moreover, although reducing $\delta_{e}$ from 0.03 to 0.01 changed the natural shedding frequency by around $0.2 \%$ only, setting $\delta_{e}$ to 0.03 affected other solution variables more severely than $0.2 \%$. So, the natural shedding frequency was not the only variable considered while choosing $\delta_{e}$.

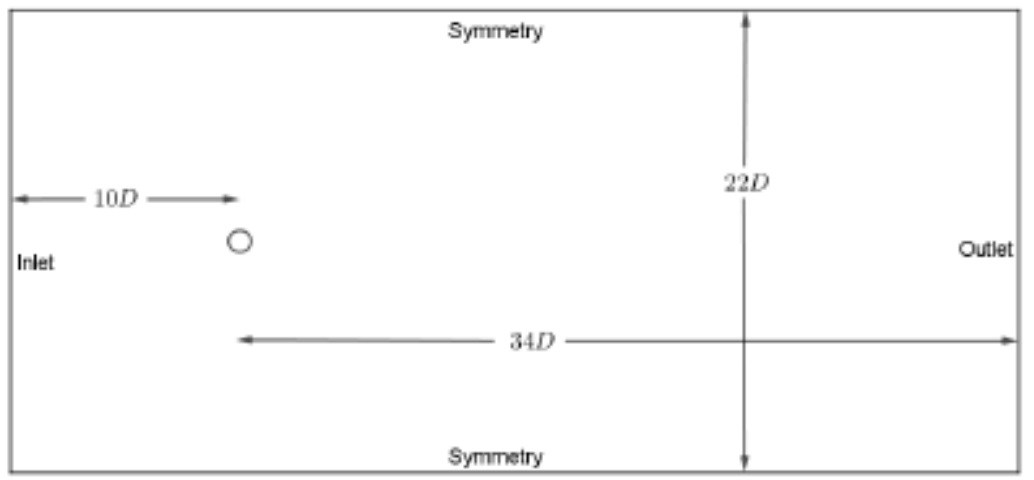

Fig. 2. A schematic of the dimensions of the computational domain referenced to the cylinder diameter

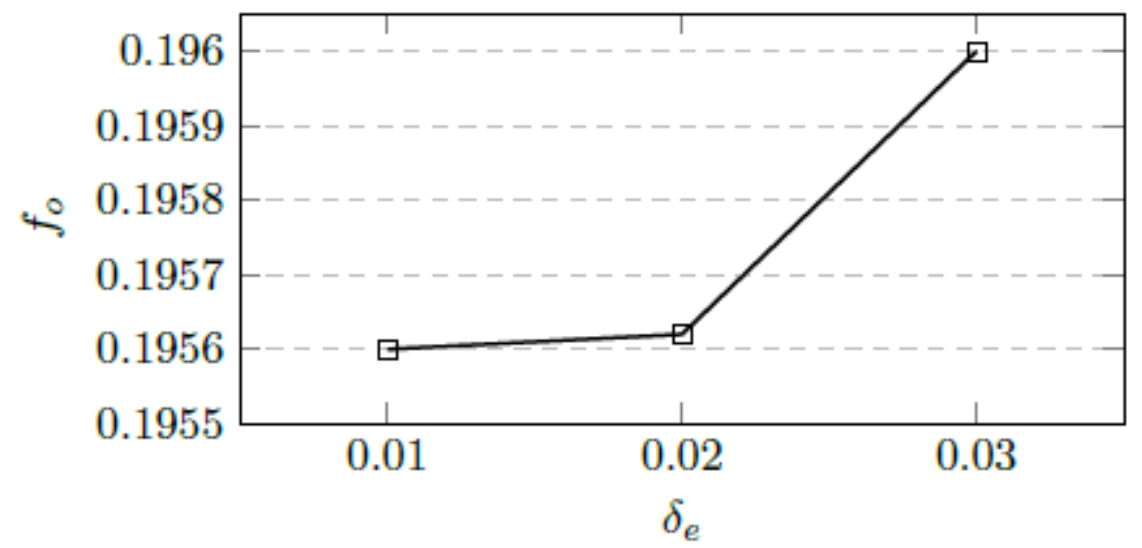

Fig. 3. Natural shedding frequency variation as a function of grid size $\boldsymbol{\delta}_{\boldsymbol{e}}$ for a stationary circular cylinder at a Reynolds number of $\mathbf{1 8 5}$

The domain's boundary conditions are set as follows. The inlet side of the rectangular domain has a velocity inlet boundary condition, where the velocity component $u$ is set to one referenced to the characteristic velocity $U_{\infty}$ and the velocity component $v$ is set to zero. The upper and lower sides have a symmetry boundary condition, along which the velocity component $v$ is set to zero and the velocity component $u$ has a zero Neumann boundary condition $(\partial u / \partial y=0)$. For the outlet side, a point is selected to have a Dirichlet boundary condition with a value of zero. This is considered as a reference pressure point. The rest of the points of the outlet side have a zero Neumann boundary condition $(\partial p / \partial x=0)$. As for the initial conditions, the domain is initialized by a zero pressure field and a value of one for both velocity components to perturb the domain in order to accelerate the vortex shedding. The non-dimensional time step $\Delta t$ for the stationary cylinder simulations is set to 0.001 with a pressure stabilization parameter $\epsilon$ set to 0.01 .

To explore the effect of the immersed boundary (IB) method along with the mapping technique, the same physical domain is used to simulate the same problem set up but using a body fitted (BF) grid. Figure 4 shows a comparison between the body fitted grid and the immersed boundary grid 
showing only a part of the cylinder. For the immersed boundary grid, grid nodes that fall inside the cylinder are colored in red, while the blue colored points represent fluid nodes. Guilmineau [37] reported a plot comparing the natural shedding frequency of a stationary circular cylinder as a function of the diameter based Reynolds number from various publications. This frequency is obtained from a Fourier transform of the lift coefficient signal. Figure 5 is a reproduction of that plot while adding the results of the current model to it. The immersed boundary case and the body fitted case are both reported. The figure shows that the results of the presented model are in acceptable agreement with experimental and numerical results of other publications. It also shows that there are not significant differences in the natural shedding frequency between the immersed boundary simulation and the body fitted simulation.
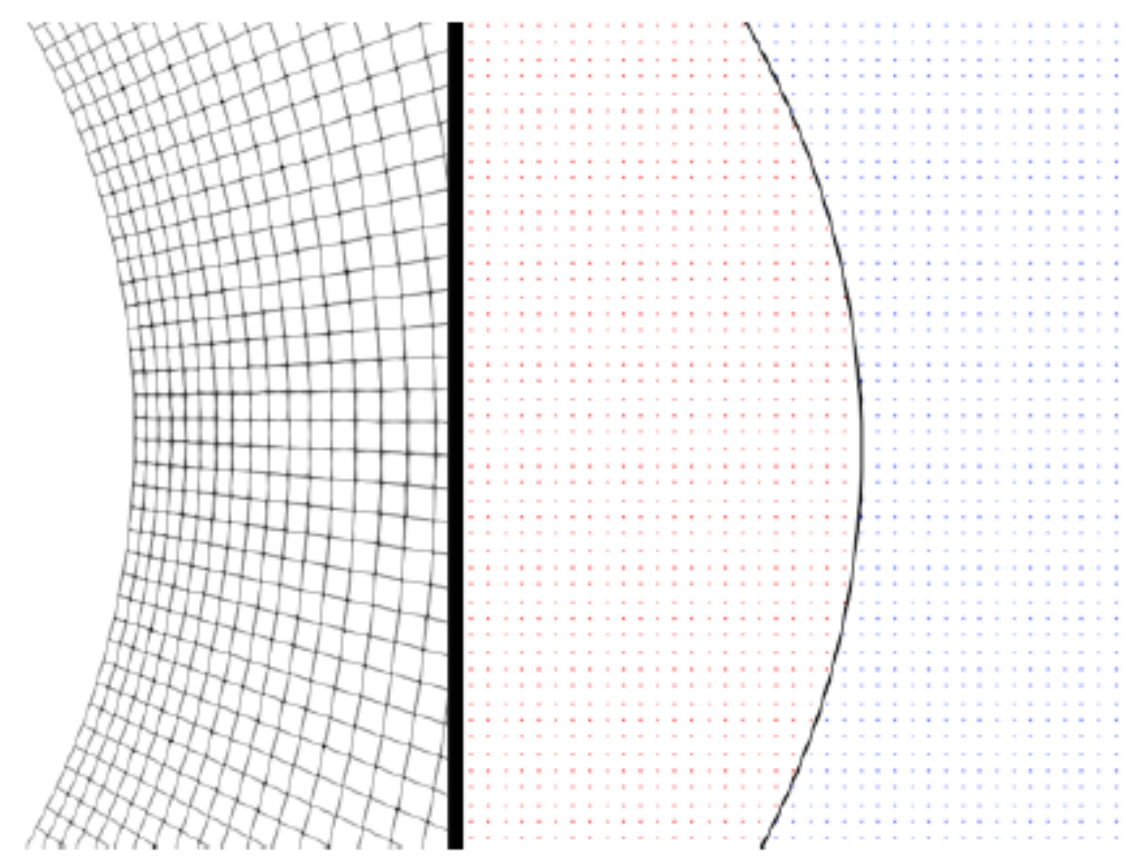

Fig. 4. A comparison between the body fitted grid (left) and the immersed boundary grid (right) for a segment of the circular cylinder

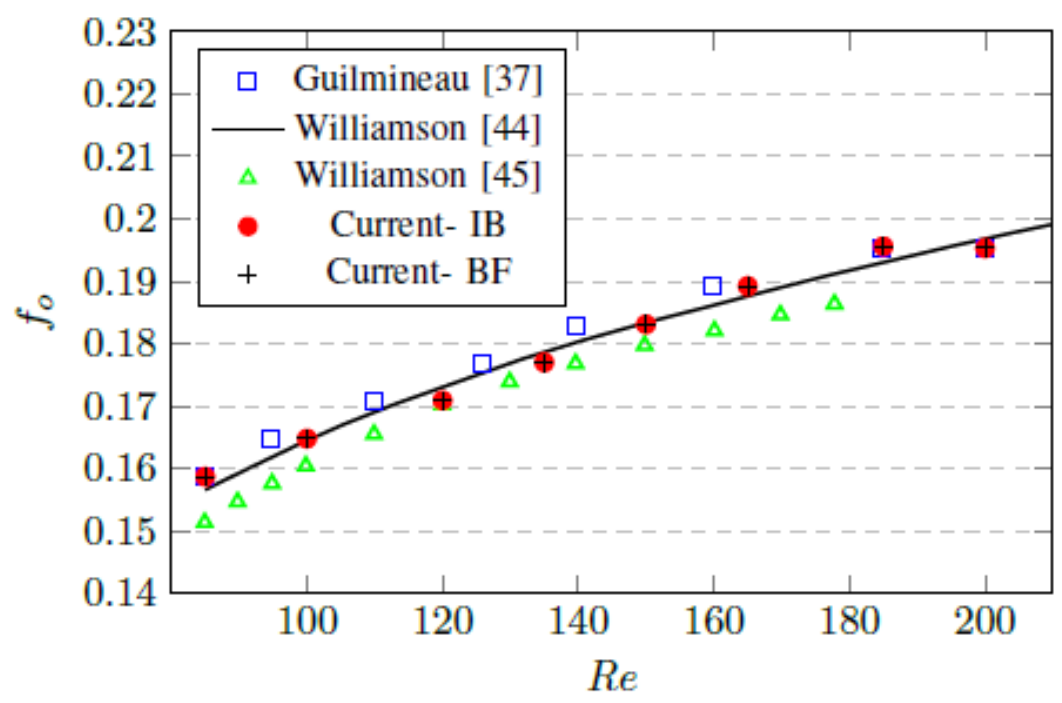

Fig. 5. The natural shedding frequency of a stationary circular cylinder as a function of the Reynolds number [44,45] 
Table 1, values relevant to the lift and drag coefficients are tabulated versus the Reynolds number for the case of the stationary cylinder using both immersed boundary and body fitted simulations. Since a non- rotating circular cylinder does not generate lift, the lift coefficient signal $\left(C_{l}\right)$ is symmetric about the zero-lift line, resulting in a zero average of the signal. The amplitude of the signal (denoted by the subscript a) is used to measure the lift signal while the mean (denoted by an upper bar sign) is used with the drag signal $\left(C_{d}\right)$ as it is not symmetric about a zero-drag line. The stress tensor as described by Eq. (17) consists of two terms, a pressure term and a viscous term. Both terms contribute differently to the generated forces, so their effect is shown separately in Table 1, where $\left(C_{l_{p}}\right)$ is the lift coefficient obtained from the pressure term in Eq. (17) and $\left(C_{l_{v}}\right)$ is the lift coefficient obtained from the viscous term. The same nomenclature is applied to the drag coefficient. Table 1 shows that that the amplitude of the lift signal is rising with the Reynolds number. This rise is caused by a rise in the amplitude of both the pressure and viscous terms in Eq. (17). On the other hand, the rise in Reynolds number raises the mean drag caused by the pressure term while it reduces the mean drag due to the viscous term. Overall, the mean drag coefficient becomes less when the flow becomes more dominant by inertia forces. The table also shows the discrepancies caused by the immersed boundary and the stress mapping relative to the body fitted case.

Table 1

Forces generated on a stationary circular cylinder at various Reynolds numbers

\begin{tabular}{llllllll}
\hline$R e$ & Type & $\left(C_{l}\right)_{a}$ & $\left(C_{l_{p}}\right)_{a}$ & $\left(C_{l_{v}}\right)_{a}$ & $\bar{C}_{d}$ & $\bar{C}_{d_{p}}$ & $\bar{C}_{d_{v}}$ \\
\hline 85 & IB & 0.265 & 0.232 & 0.038 & 1.438 & 1.086 & 0.352 \\
& BF & 0.288 & 0.252 & 0.042 & 1.436 & 1.064 & 0.371 \\
100 & IB & 0.326 & 0.289 & 0.043 & 1.420 & 1.100 & 0.322 \\
& BF & 0.351 & 0.310 & 0.047 & 1.418 & 1.080 & 0.342 \\
120 & IB & 0.400 & 0.358 & 0.047 & 1.414 & 1.124 & 0.291 \\
& BF & 0.425 & 0.380 & 0.052 & 1.403 & 1.092 & 0.311 \\
135 & IB & 0.452 & 0.408 & 0.050 & 1.412 & 1.140 & 0.272 \\
& BF & 0.477 & 0.429 & 0.055 & 1.398 & 1.105 & 0.293 \\
150 & IB & 0.501 & 0.456 & 0.052 & 1.414 & 1.157 & 0.257 \\
& BF & 0.527 & 0.477 & 0.057 & 1.396 & 1.118 & 0.278 \\
165 & IB & 0.548 & 0.501 & 0.054 & 1.418 & 1.174 & 0.243 \\
& BF & 0.574 & 0.522 & 0.059 & 1.395 & 1.131 & 0.265 \\
185 & IB & 0.606 & 0.558 & 0.056 & 1.425 & 1.197 & 0.228 \\
& BF & 0.633 & 0.579 & 0.061 & 1.398 & 1.148 & 0.250 \\
200 & IB & 0.647 & 0.598 & 0.056 & 1.429 & 1.211 & 0.218 \\
& BF & 0.674 & 0.619 & 0.063 & 1.402 & 1.162 & 0.240 \\
\hline
\end{tabular}

Figure 6 shows a comparison of the pressure contours between the body fitted case, shown on the upper part of the figure, and the immersed boundary case on the bottom side of the figure for a stationary cylinder and a Reynolds number of 100 . The contours were generated at a moment in time after the start of the vortices shedding in both simulations. The figure shows a great agreement between the contours of both cases. In the lower sub-figure the contours inside the cylinder are suppressed as they present no physical meaning. However, these inside nodes are shown in Figure 7 having a zero velocity (the velocity of the stationary cylinder). Figure 7 shows velocity surfaces of both the body fitted and immersed boundary cases. Figure 8 shows the distribution of the cycle averaged pressure coefficient along the surface of the cylinder at a Reynolds number of 100 for both the immersed boundary and body fitted simulations. Both results are compared to the results of Park et al., [46]. 


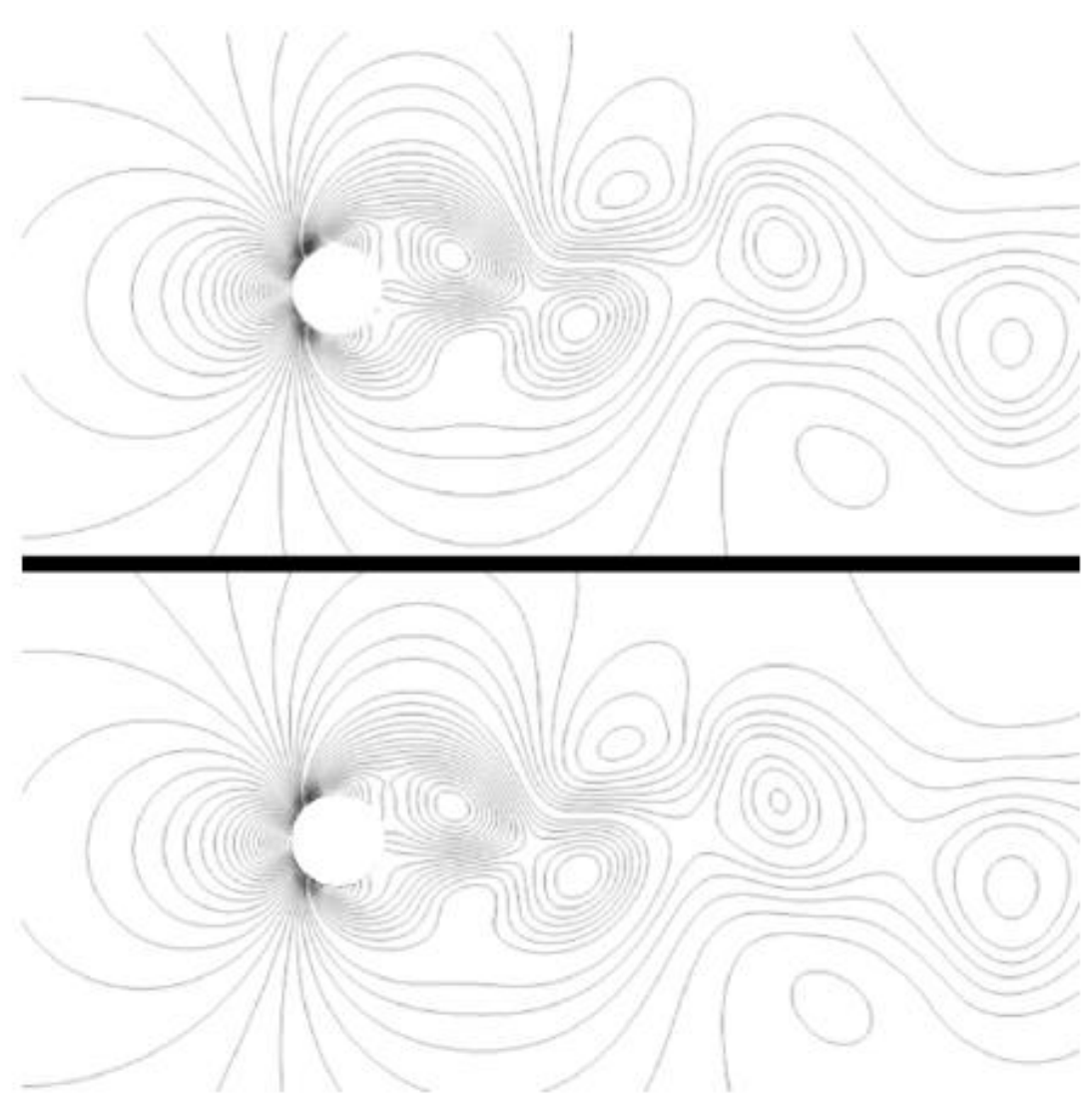

Fig. 6. A pressure contour comparison between the body fitted simulation (top) and the immersed boundary simulation (bottom) for a stationary circular cylinder at a Reynolds number of 100

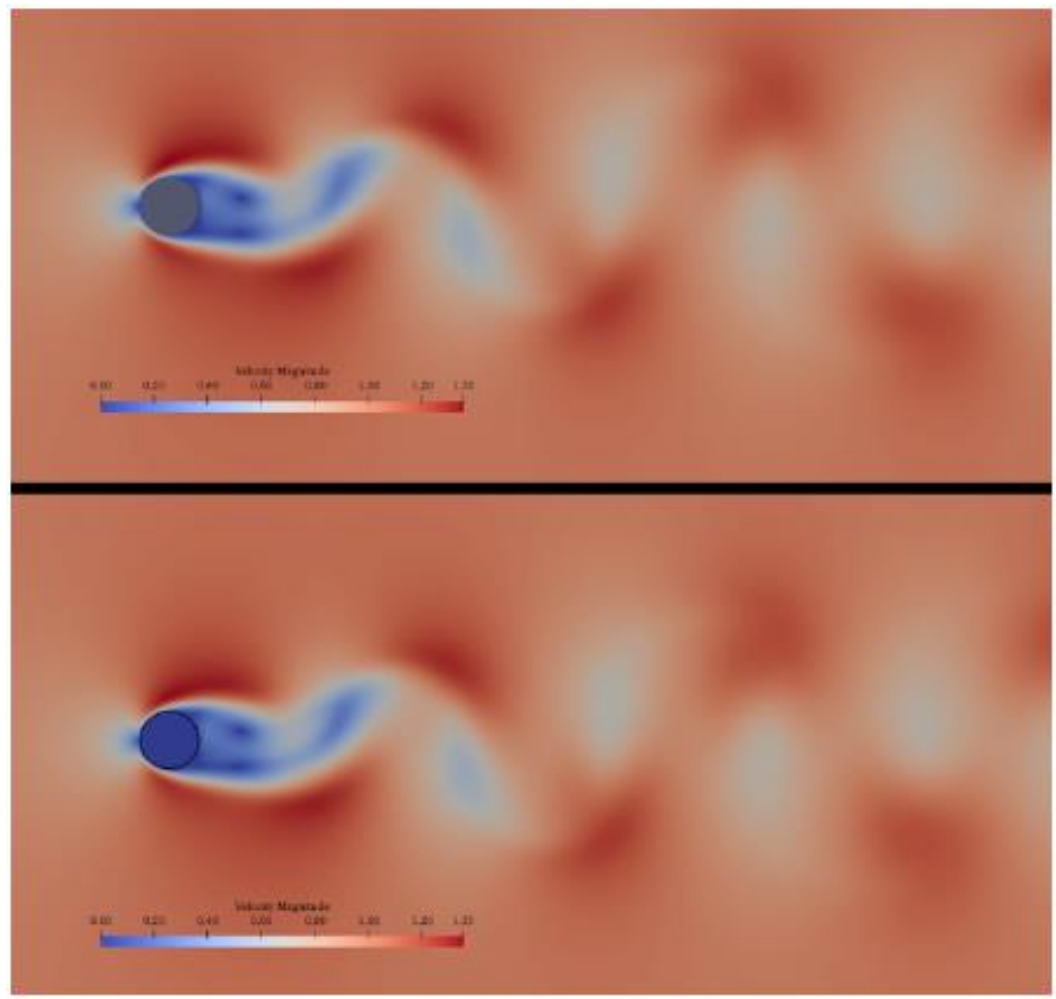

Fig. 7. A velocity field comparison between the body fitted simulation (top) and the immersed boundary simulation (bottom) for a stationary circular cylinder at a Reynolds number of 100 
The cycle averaged pressure distribution along the cylinder's surface is symmetric. So, only half of the cylinder is shown in Figure 8 with the angle $\theta$ being zero at the leading stagnation point. The figure shows great accordance between the current model and previous publications. Mapping the pressure field onto the cylinder resulted in some noise in the pressure coefficient distribution. Such a noise is directly proportional to the grid size. In this work, the magnitude of the Cauchy stress tensor is defined as $\sqrt{\sigma: \sigma}$, where : is the double contraction tensor operator. The cycle averaged stress magnitude is plotted in Figure 9 for both the immersed boundary and the body fitted simulations. The plot shows a great accordance between the two models and hence strengthening the reliability of the presented immersed boundary model.

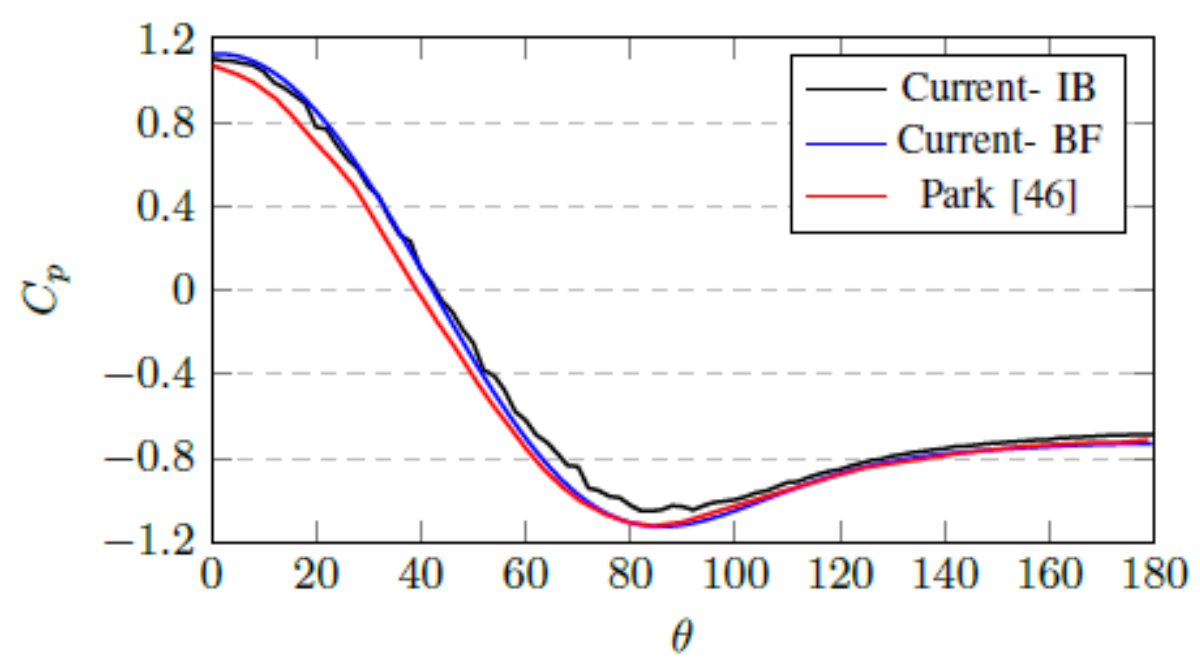

Fig. 8. Cycle averaged pressure coefficient distribution around the cylinder's surface at a Reynolds number of 100

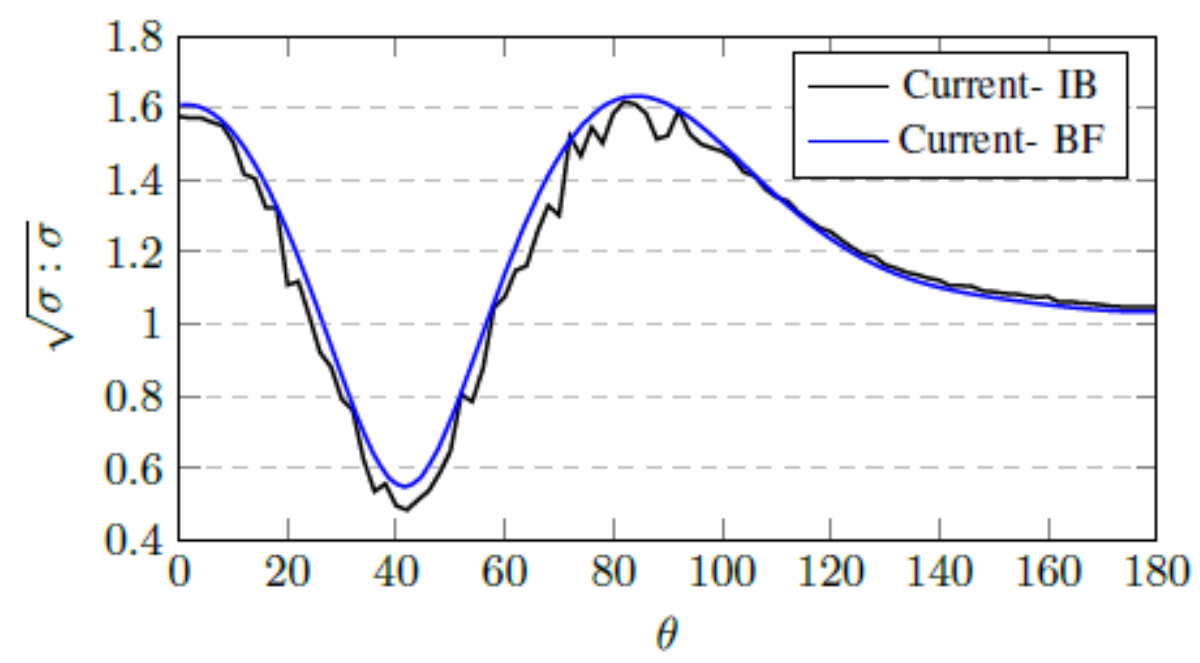

Fig. 9. Cycle averaged Cauchy stress magnitude distribution around the cylinder's surface at a Reynolds number of 100

\subsection{Transverse Flow Over a Vertically Oscillating Circular Cylinder}

In this section, the presented model is validated against experimental and numerical data in the literature for vertically as well as horizontally oscillating circular cylinders. The flow domain is the 
exact to that of the stationary cylinder with the same boundary conditions except that of the solid nodes which get the velocity of the oscillating cylinder.

$\chi=-E_{a} \sin \left(2 \pi f_{e} t\right)$

The case of an oscillating circular cylinder is defined, beside the flow Reynolds number, by the excitation frequency $f_{e}$ and the excitation amplitude $E_{a}$ as shown in Eq. (20), where $\chi$ is the direction of motion of the cylinder ( $y$ for a vertically oscillating cylinder, and $x$ for a horizontally oscillating cylinder). $\chi$ and $E_{a}$ are referenced to the cylinder diameter $D$. The excitation frequency $f_{e}$ is often referenced to the natural shedding frequency $f_{o}$ of the stationary correspondent of the oscillating cylinder. The frequency ratio $f_{r}$ is defined as the ratio between the excitation frequency $f_{e}$ and the natural shedding frequency $f_{o}$ of the stationary cylinder at the same Reynolds number.

$f_{r}=f_{e} / f_{o}$

The lock-in phenomenon of the oscillating circular cylinder is defined as a state where the frequency of vortices shedding for an oscillating cylinder is locked to the excitation frequency [23]. Pham et al., [39] simulated several cases of vertically oscillating cylinders that fall inside the lock-in zone on the excitation amplitude - excitation frequency plane. Three different frequency ratios will be simulated and compared to the results of [39] for validation. For a Reynolds number of 185 and an excitation amplitude of 0.2 , the frequency ratios $f_{r}$ of $0.8,1.0$, and 1.1 will be simulated using the immersed boundary model. Table 2 shows a comparison of the frequency of vortices shedding $f_{S}$, the root mean squared lift coefficient $\left(C_{l}\right)_{r}$, and the mean drag coefficient $\bar{C}_{d}$ between the current immersed boundary model and the results of [39] for a Reynolds number of 185 and an excitation amplitude of 0.2 . The table shows a great agreement with the published results, while noting that the values in the " $f_{s}$ " column of the current model are exactly the product of the frequency ratio $f_{r}$ and the natural shedding frequency $f_{o}$ at a Reynolds number of 185 as shown in Figure 5 by a value of 0.1956 . This is in accordance with the fact that the three simulated points lie in the lock-in zone.

\section{Table 2}

A comparison between shedding frequency, root mean squared lift coefficient, and mean drag coefficient at various excitation frequency ratios between the current model and the results of Pham et al., [39] at a Reynolds number of 185 and an excitation amplitude of 0.2

\begin{tabular}{lllllll}
\hline$f_{r}$ & Current- IB & \multicolumn{5}{c}{ Pham et al., [39] } \\
\cline { 2 - 7 } & $f_{s}$ & $\left(C_{l}\right)_{r}$ & $\bar{C}_{d}$ & $f_{s}$ & $\left(C_{l}\right)_{r}$ & $\bar{C}_{d}$ \\
\hline 0.8 & 0.1564 & 0.1009 & 1.31 & 0.1533 & 0.101 & 1.24 \\
1.0 & 0.1956 & 0.443 & 1.653 & 0.1933 & 0.424 & 1.532 \\
1.1 & 0.2151 & 0.8504 & 1.4304 & 0.2133 & 0.866 & 1.388 \\
\hline
\end{tabular}

To demonstrate the effect of both pressure and viscous terms of Cauchy stress tensor, the values of the pressure and viscous components of both the lift and drag coefficients are tabulated in Table 3. Again, lift coefficient related values are tabulated as root mean squared values, while drag coefficient related values are tabulated as mean values. Figure 10 shows the temporal variation of the lift and drag coefficients signals for the frequency ratios of $0.8,1.0$, and 1.1 , respectively. The $x$ axis of each of these figures has the time normalized by its own excitation period, such that the axis represents cycles of excitation. In this way, all the plots share the same $x$-axis. In Figure 10 , the black curves represent the lift coefficient and the red curves represent the drag coefficient. For the top and middle plots of Figure 10, the excitation frequency did not surpass the natural shedding frequency of 
the stationary cylinder. For such cases, the generated forces signals are purely sinusoidal with their shedding frequencies equal to the excitation frequencies. However, when the excitation frequency exceeds the natural shedding frequency of the stationary cylinder, the generated forces become sinusoidal but with a limit-cycle behaviour. This means that the signal is composed of patches of sinusoidal signals that are repeated with a frequency that is less than the primary shedding frequency. The latter is still equal to the excitation frequency as long as the excitation frequency and amplitude remain in the lock-in zone.

Table 3

Forces generated on an oscillating circular cylinder at various excitation frequencies for a Reynolds number of 185 and an excitation amplitude of 0.2

\begin{tabular}{lllllll}
\hline$f_{r}$ & $\left(C_{l}\right)_{r}$ & $\left(C_{l_{p}}\right)_{r}$ & $\left(C_{l_{v}}\right)_{r}$ & $\bar{C}_{d}$ & $\bar{C}_{d_{p}}$ & $\bar{C}_{d_{v}}$ \\
\hline 0.8 & 0.1009 & 0.1160 & 0.0152 & 1.31 & 1.0904 & 0.2196 \\
1.0 & 0.4430 & 0.4393 & 0.0405 & 1.653 & 1.3954 & 0.2575 \\
1.1 & 0.8504 & 0.7624 & 0.1083 & 1.4304 & 1.2022 & 0.2282 \\
\hline
\end{tabular}
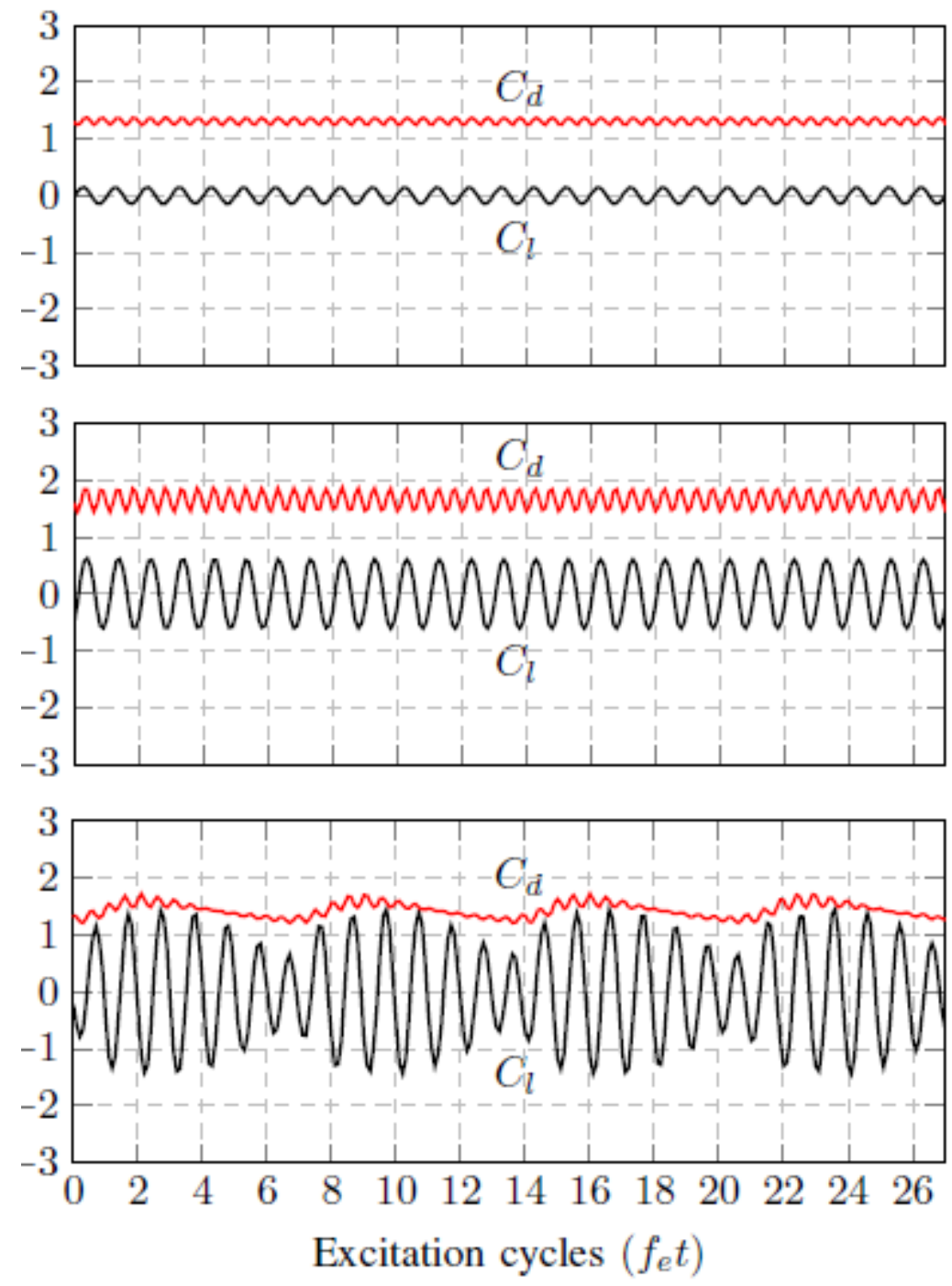

Fig. 10. Lift and drag coefficient signals for a Reynolds number of $\mathbf{1 8 5}$ and an excitation amplitude of $\mathbf{0 . 2}$. Top: $\boldsymbol{f}_{r}=\mathbf{0 . 8}$, middle: $f_{r}=1.0$, bottom: $f_{r}=1.1$ 


\subsection{A Horizontally Oscillating Circular Cylinder in a Stagnant Fluid}

Dutsch et al., [47] presented some experimental measurements for a horizontally oscillating circular cylinder in a stagnant fluid. The measurements included vertical velocity profiles at multiple longitudinal locations. Since no free stream velocity is present, the definition of the Reynolds number is altered. In this context, the Reynolds number will be based on the maximum oscillation velocity of the cylinder $u_{\max }=2 \pi f_{e} E_{a}$. So, the definition of the Reynolds number becomes $R e=\rho u_{\max } D / \mu$. The Keulegan-Carpenter number $K C$ is defined as $u_{\max } /\left(f_{e} D\right)$. For a Reynolds number of 100 and an excitation amplitude $E_{a}$ of $5 / 2 \pi$ (corresponding to a Keulegan-Carpenter number of 5 ), Figures (11-14) shows the vertical distributions of both velocity components at four different longitudinal locations. The locations are at $x=-0.6, x=0, x=0.6$, and $x=1.2$ with experimental measurements provided by Dutsch et al., [47] and numerical results provided by Uzunoglu et al., [48]. The figures show a good agreement with both the experimental data and the numerical results. Figure (15) shows a comparison with [47] and [48] regarding the variation of the in-line (drag) force during the excitation cycle of the cylinder. Again, the results are quite close.

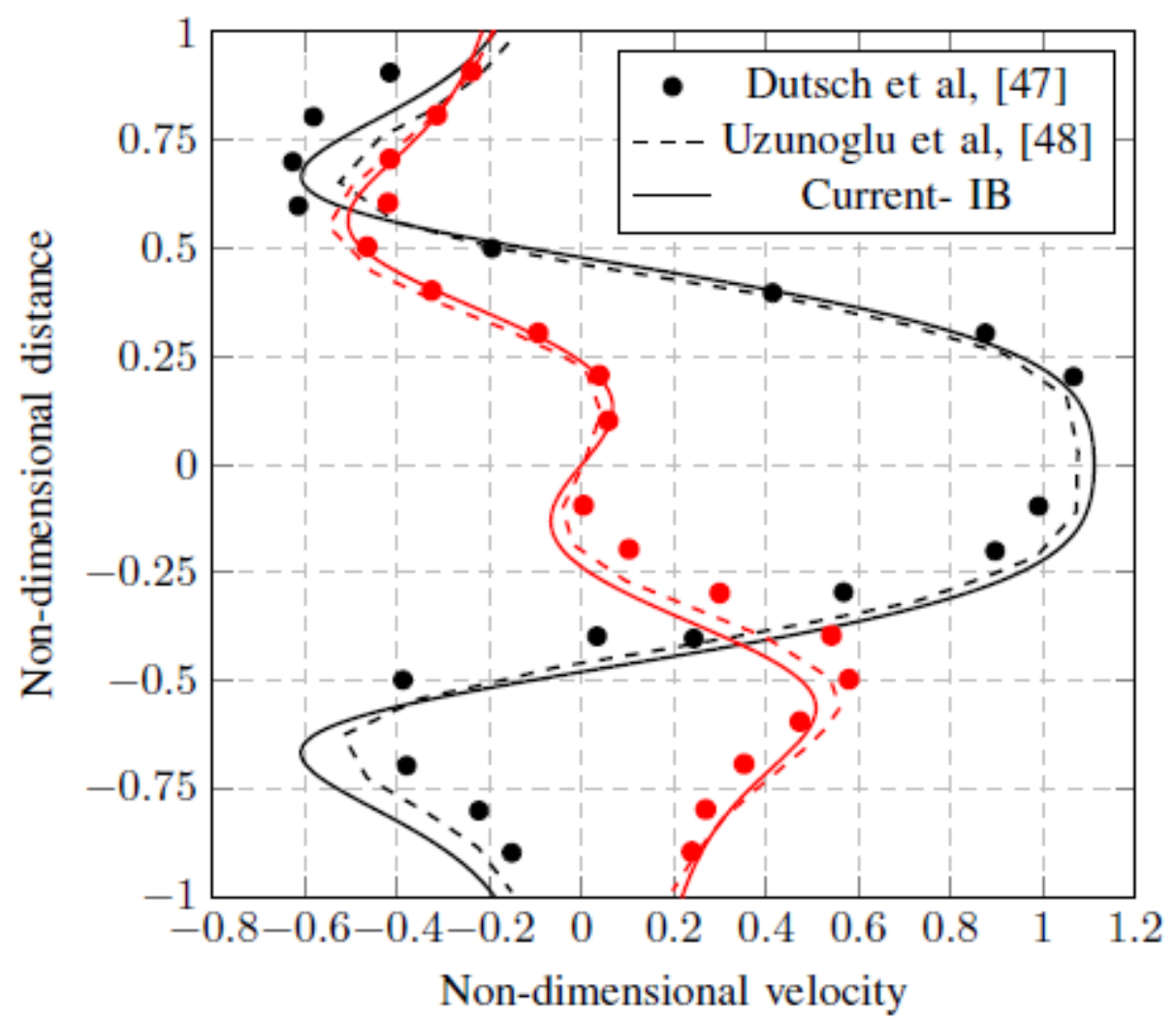

Fig. 11. The velocity distribution at a longitudinal location of $\boldsymbol{x}=-\mathbf{0 . 6}$ in the middle of an excitation cycle for a Reynolds number of $\mathbf{1 0 0}$ and $\boldsymbol{K} \boldsymbol{C}$ of $\mathbf{5}$. Black: $\boldsymbol{u}$, Red: $\boldsymbol{v}$ 


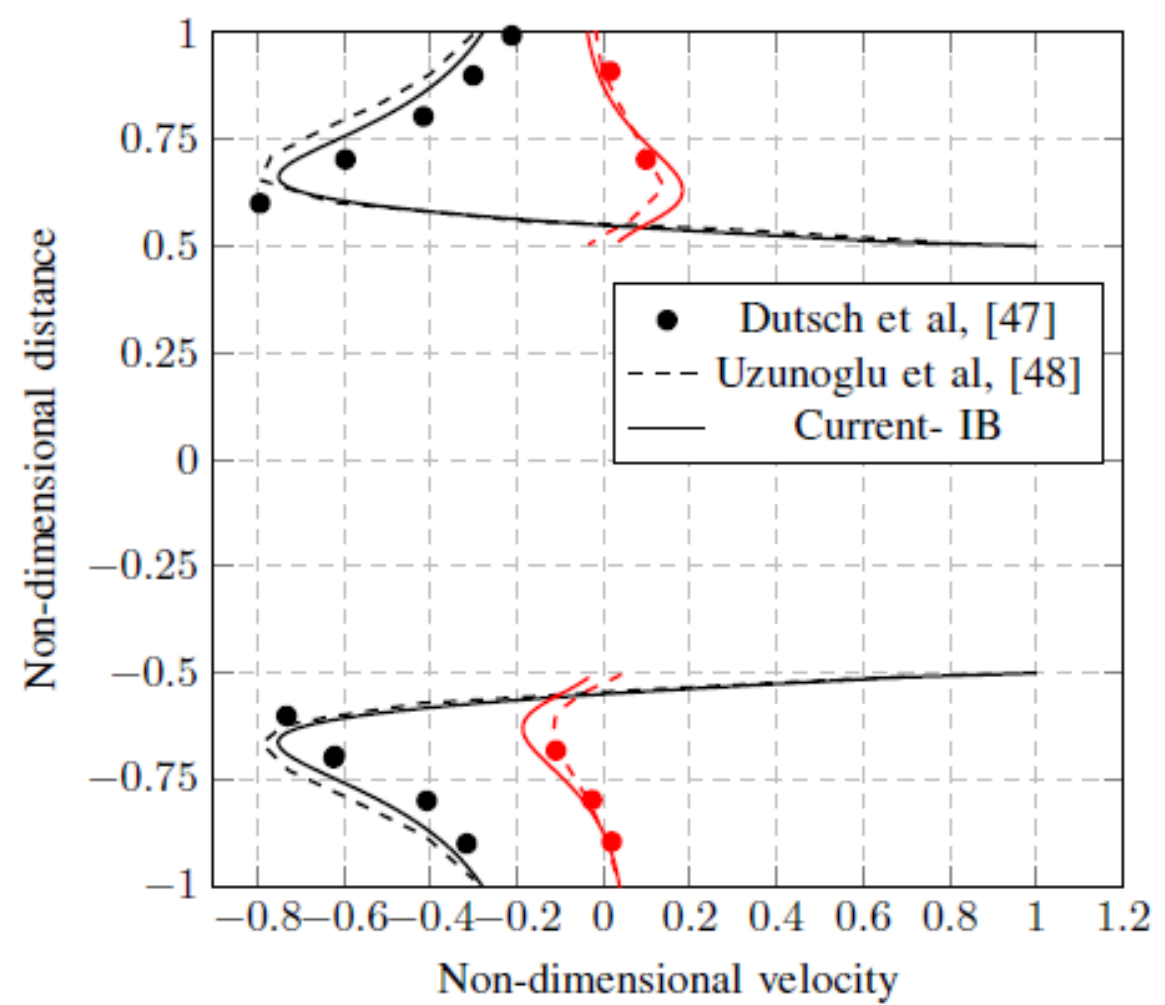

Fig. 12. The velocity distribution at a longitudinal location of $\boldsymbol{x}=\mathbf{0}$ in the middle of an excitation cycle for a Reynolds number of $\mathbf{1 0 0}$ and $\boldsymbol{K} \boldsymbol{C}$ of $\mathbf{5}$. Black: $\boldsymbol{u}$, Red: $\boldsymbol{v}$



Fig. 13. The velocity distribution at a longitudinal location of $\boldsymbol{x}=\mathbf{0 . 6}$ in the middle of an excitation cycle for a Reynolds number of 100 and KC of 5. Black: u, Red: v 


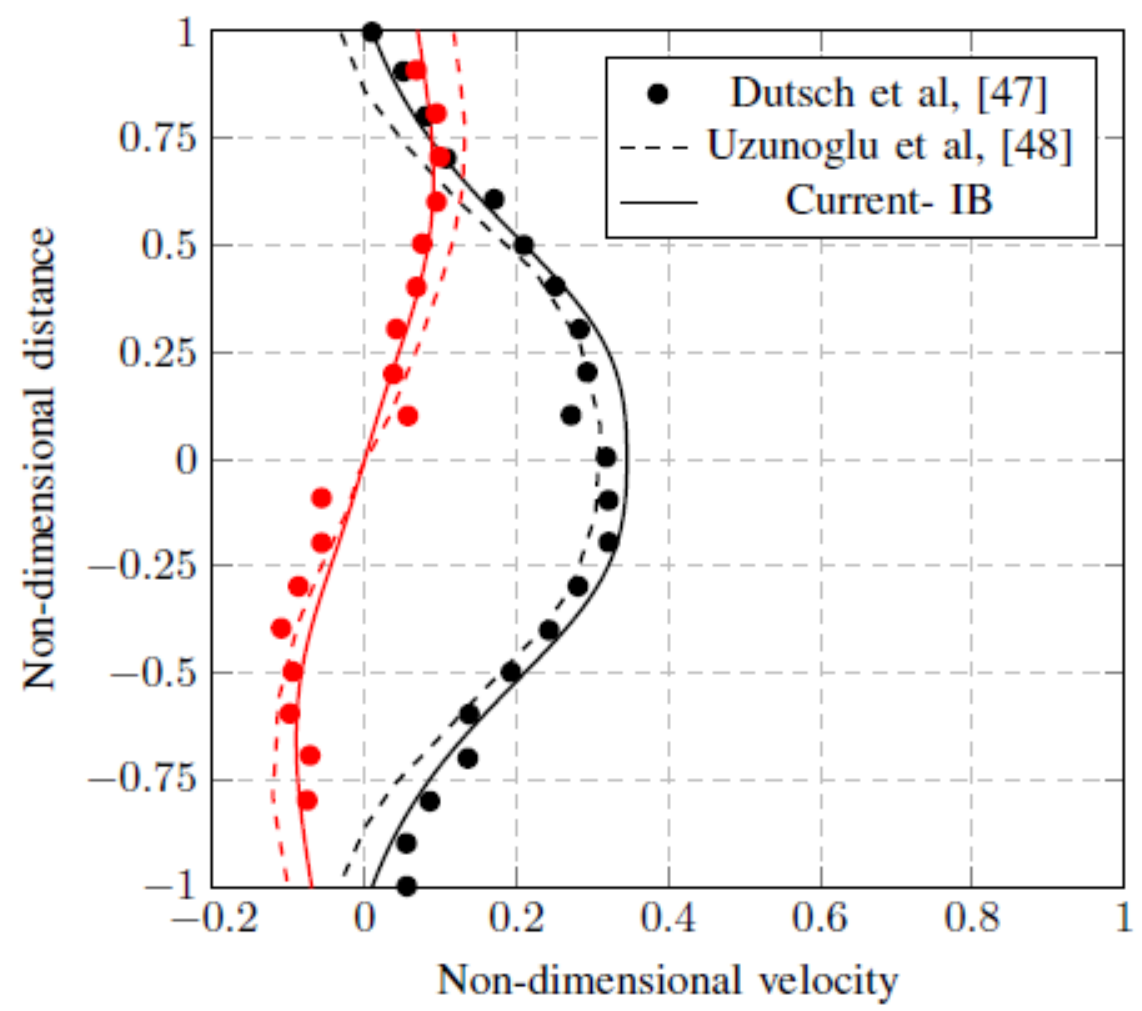

Fig. 14. The velocity distribution at a longitudinal location of $x=1.2$ in the middle of an excitation cycle for a Reynolds number of 100 and KC of 5. Black: u, Red: v

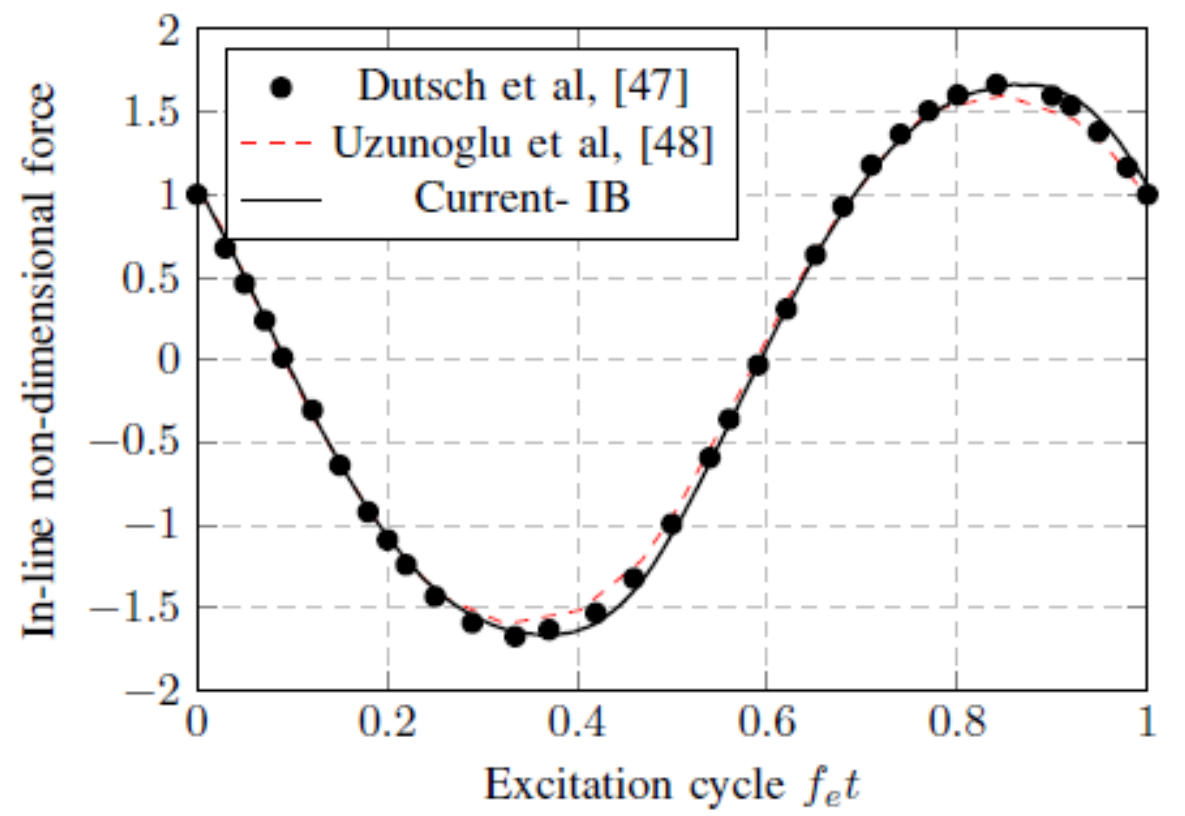

Fig. 15. In-line force variation during an excitation cycle

\section{Summary and Conclusions}

In this work, an immersed boundary technique based on stress mapping is presented. The technique depends on modeling the solid regions as infinitely viscous fluid regions to ensure a proper transfer of momentum from the moving body to the flow. The stresses acting on the moving body are calculated using a mapping technique where each vertex of the body is regarded as a virtual fluid 
node whose stress is interpolated from the fluid nodes of its enclosing grid element. The mapped stresses are then integrated over the body's contour creating a well-defined proper contour of integration. The presented model was validated against published experimental measurements and numerical results for a stationary cylinder, a transversely oscillating cylinder, and a longitudinally oscillating cylinder in stagnant flow. The validation showed good agreement with the literature.

\section{Declaration of Interests}

The authors report no conflict of interest.

\section{References}

[1] Baker, Timothy J. "Mesh movement and metamorphosis." Engineering with Computers 18, no. 3 (2002): 188-198. https://doi.org/10.1007/s003660200017

[2] Liao, Guojun, and Jiaxing Xue. "Moving meshes by the deformation method." Journal of computational and applied mathematics 195, no. 1-2 (2006): 83-92. https://doi.org/10.1016/i.cam.2005.07.022

[3] Shontz, Suzanne M., and Stephen A. Vavasis. "A Mesh Warping Algorithm Based on Weighted Laplacian Smoothing." In IMR, pp. 147-158. 2003.

[4] Carey, Graham F. Computational grids: generations, adaptation \& solution strategies. CRC Press, 1997.

[5] Liseikin, Vladimir D. Grid generation methods. Vol. 1. Berlin: Springer, 1999. https://doi.org/10.1007/978-3-66203949-6 1

[6] Jafari, A., and N. Ashgriz. "Numerical techniques for free surface flows: Interface capturing and interface tracking." Encyclopedia of Microfluidics and Nanofluidics (2015): 2458-2479. https://doi.org/10.1007/978-1-4614$\underline{5491-51139}$

[7] Harlow, F. H., and J. E. Welch. "A domain decomposition method for incompressible viscous flow of fluid with free surface." Physics Fluids 8 (1965): 2182-2189. https://doi.org/10.1063/1.1761178

[8] Nichols, B. D., C. W. Hirt, and R. S. Hotchkiss. "Volume of fluid (VOF) method for the dynamics of free boundaries." Journal of Computational Physics 39, no. 1 (1981): 201-225. https://doi.org/10.1016/00219991(81)90145-5

[9] Fedkiw, Stanley Osher Ronald, and Stanley Osher. "Level set methods and dynamic implicit surfaces." Surfaces 44, no. 77 (2002): 685.

[10] Udaykumar, H. S., Rajat Mittal, and Wei Shyy. "Computation of solid-liquid phase fronts in the sharp interface limit on fixed grids." Journal of computational physics 153, no. 2 (1999): 535-574. https://doi.org/10.1006/jcph.1999.6294

[11] Francois, Marianne, and Wei Shyy. "Computations of drop dynamics with the immersed boundary method, part 1: numerical algorithm and buoyancy-induced effect." Numerical Heat Transfer: Part B: Fundamentals 44, no. 2 (2003): 101-118. https://doi.org/10.1080/713836347

[12] Bagabir, A., A. Balabel, and W. A. El-Askary. "On the Dynamics of Shock-Interface Interaction using the Level Set Method." CFD Letters 2, no. 4 (2010): 176-196.

[13] Duo-Xing, Yang, and Zhang De-liang. "Simulating Multiphase Flows in Porous Media with High Order CE/SE Method." CFD Letters 3, no. 1 (2011): 1-17.

[14] Muldoon, Frank, and Sumanta Acharya. "Mass Conservation in the Immersed Boundary Method." In Fluids Engineering Division Summer Meeting, vol. 41987, pp. 411-419. 2005. https://doi.org/10.1115/FEDSM2005-77301

[15] Mittal, Rajat, and Gianluca laccarino. "Immersed boundary methods." Annu. Rev. Fluid Mech. 37 (2005): $239-261$. https://doi.org/10.1146/annurev.fluid.37.061903.175743

[16] Peskin, Charles S. "The fluid dynamics of heart valves: experimental, theoretical, and computational methods." Annual review of fluid mechanics 14, no. 1 (1982): $235-259$. https://doi.org/10.1146/annurev.fl.14.010182.001315

[17] Fauci, Lisa J., and Amy McDonald. "Sperm motility in the presence of boundaries." Bulletin of mathematical biology 57, no. 5 (1995): 679-699. https://doi.org/10.1016/0092-8240(95)00022-I

[18] Tutkun, Bulent, and Firat Oguz Edis. "An implementation of the direct-forcing immersed boundary method using GPU power." Engineering Applications of Computational Fluid Mechanics 11, no. 1 (2017): 15-29. https://doi.org/10.1080/19942060.2016.1236749

[19] Handler, R., and L. Sirovich. "Modeling a no-slip surface with an external force field." J. Comput. Phys 105 (1993): 354. https://doi.org/10.1006/icph.1993.1081 
[20] Angot, Philippe, Charles-Henri Bruneau, and Pierre Fabrie. "A penalization method to take into account obstacles in incompressible viscous flows." Numerische Mathematik 81, no. $4 \quad$ (1999): $497-520$. https://doi.org/10.1007/s002110050401

[21] Mohd-Yusof, Jamaludin. "Combined immersed-boundary/B-spline methods for simulations of flow in complex geometries." Center for turbulence research annual research briefs 161, no. 1 (1997): 317-327.

[22] Ferziger, Joel H., Milovan Perić, and Robert L. Street. Computational methods for fluid dynamics. Vol. 3. Berlin: springer, 2002. https://doi.org/10.1007/978-3-642-56026-2

[23] Clarke, D. Keith, M. D. Salas, and H. A. Hassan. "Euler calculations for multielement airfoils using Cartesian grids." AIAA journal 24, no. 3 (1986): 353-358. https://doi.org/10.2514/3.9273

[24] Salih, Sinan Q., Mohammed Suleman Aldlemy, Mohammad Rasidi Rasani, A. K. Ariffin, Tuan Mohammad Yusoff Shah Tuan Ya, Nadhir Al-Ansari, Zaher Mundher Yaseen, and Kwok-Wing Chau. "Thin and sharp edges bodies-fluid interaction simulation using cut-cell immersed boundary method." Engineering Applications of Computational Fluid Mechanics 13, no. 1 (2019): 860-877. https://doi.org/10.1080/19942060.2019.1652209

[25] Brezzi, Franco, and Juhani Pitkäranta. "On the stabilization of finite element approximations of the Stokes equations." In Efficient solutions of elliptic systems, pp. 11-19. Vieweg+ Teubner Verlag, Wiesbaden, 1984. https://doi.org/10.1007/978-3-663-14169-3 2

[26] Franca, Leopoldo P., Guillermo Hauke, and Arif Masud. "Revisiting stabilized finite element methods for the advective-diffusive equation." Computer Methods in Applied Mechanics and Engineering 195, no. 13-16 (2006): 1560-1572. https://doi.org/10.1016/i.cma.2005.05.028

[27] Hughes, Thomas JR, Leopoldo P. Franca, and Gregory M. Hulbert. "A new finite element formulation for computational fluid dynamics: VIII. The Galerkin/least-squares method for advective-diffusive equations." Computer methods in applied mechanics and engineering 73, no. 2 (1989): $173-189$. https://doi.org/10.1016/0045-7825(89)90111-4

[28] Ali, Karim M., and Amr Guaily. "Dual perspective method for solving the point in a polygon problem." arXiv preprint arXiv:2012.05001 (2020).

[29] Shen, Jie. "On pressure stabilization method and projection method for unsteady Navier-Stokes equations." In Advances in Computer Methods for Partial Differential Equations. 1992.

[30] Shepard, Donald. "A two-dimensional interpolation function for irregularly-spaced data." In Proceedings of the 1968 23rd ACM national conference, pp. 517-524. 1968. https://doi.org/10.1145/800186.810616

[31] Bishop, Richard Evelyn Donohue, and A. Y. Hassan. "The lift and drag forces on a circular cylinder oscillating in a flowing fluid." Proceedings of the Royal Society of London. Series A. Mathematical and Physical Sciences 277 , no. 1368 (1964): 51-75. https://doi.org/10.1098/rspa.1964.0005

[32] Koopmann, G. H. "The vortex wakes of vibrating cylinders at low Reynolds numbers." Journal of Fluid Mechanics 28, no. 3 (1967): 501-512. https://doi.org/10.1017/S0022112067002253

[33] Williamson, Charles HK, and Anatol Roshko. "Vortex formation in the wake of an oscillating cylinder." Journal of fluids and structures 2, no. 4 (1988): 355-381. https://doi.org/10.1016/S0889-9746(88)90058-8

[34] Tezduyar, Tayfun E., Kenji Takizawa, Creighton Moorman, Samuel Wright, and Jason Christopher. "Space-time finite element computation of complex fluid-structure interactions." International Journal for Numerical Methods in Fluids 64, no. 10-12 (2010): 1201-1218. https://doi.org/10.1002/fld.2221

[35] Lu, X-Y., and C. Dalton. "Calculation of the timing of vortex formation from an oscillating cylinder." Journal of Fluids and Structures 10, no. 5 (1996): 527-541. https://doi.org/10.1006/jfls.1996.0035

[36] Anagnostopoulos, Petros. "Numerical study of the flow past a cylinder excited transversely to the incident stream. Part 1: Lock-in zone, hydrodynamic forces and wake geometry." Journal of Fluids and Structures 14, no. 6 (2000): 819-851. https://doi.org/10.1006/jfls.2000.0302

[37] Guilmineau, Emmanuel, and P. Queutey. "A numerical simulation of vortex shedding from an oscillating circular cylinder." Journal of Fluids and Structures 16, no. 6 (2002): 773-794. https://doi.org/10.1006/jfls.2002.0449

[38] Placzek, Antoine, Jean-François Sigrist, and Aziz Hamdouni. "Numerical simulation of an oscillating cylinder in a cross-flow at low Reynolds number: Forced and free oscillations." Computers \& Fluids 38, no. 1 (2009): 80-100. https://doi.org/10.1016/i.compfluid.2008.01.007

[39] Anh-Hung, Pham, Lee Chang-Yeol, Seo Jang-Hoon, Chun Ho-Hwan, Kim Hee-Jung, Hyun-Sik Yoon, Dong-Woo Park, and II-Ryong Park. "Laminar flow past an oscillating circular cylinder in cross flow." Journal of Marine Science and Technology 18, no. 3 (2010): 5. https://doi.org/10.51400/2709-6998.1881

[40] Shrivastava, Mukul, Amit Agrawal, and Atul Sharma. "A novel level set-based immersed-boundary method for CFD simulation of moving-boundary problems." Numerical Heat Transfer, Part B: Fundamentals 63, no. 4 (2013): 304326. https://doi.org/10.1080/10407790.2013.756258 
[41] Mustto, Angelo A., and Gustavo CR Bodstein. "Subgrid-scale modeling of turbulent flow around circular cylinder by mesh-free vortex method." Engineering applications of computational fluid mechanics 5, no. 2 (2011): 259-275. https://doi.org/10.1080/19942060.2011.11015369

[42] Sheikh, Nadeem Ahmed, and Shehryar Manzoor. "Numerical Simulation of Phase Map Shift of Vibrating Cylinder at Low Reynolds Number." CFD Letters 5, no. 3 (2013): 97-107.

[43] Persillon, Helene, and Marianna Braza. "Physical analysis of the transition to turbulence in the wake of a circular cylinder by three-dimensional Navier-Stokes simulation." Journal of Fluid Mechanics 365 (1998): 23-88. https://doi.org/10.1017/S0022112098001116

[44] Williamson, Charles HK. "Defining a universal and continuous Strouhal-Reynolds number relationship for the laminar vortex shedding of a circular cylinder." The Physics of fluids 31, no. 10 (1988): 2742-2744. https://doi.org/10.1063/1.866978

[45] Williamson, Charles HK. "Oblique and parallel modes of vortex shedding in the wake of a circular cylinder at low Reynolds numbers." Journal of $\quad$ Fluid $\quad$ Mechanics $206 \quad$ (1989): $579-627$. https://doi.org/10.1017/S0022112089002429

[46] Park, Jeongyoung, Kiyoung Kwon, and Haecheon Choi. "Numerical solutions of flow past a circular cylinder at Reynolds numbers up to 160." KSME international Journal 12, no. 6 (1998): 1200-1205. https://doi.org/10.1007/BF02942594

[47] Dütsch, H., F. Durst, S. Becker, and H. Lienhart. "Low-Reynolds-number flow around an oscillating circular cylinder at low Keulegan-Carpenter numbers." Journal of Fluid Mechanics 360 (1998): $249-271$. https://doi.org/10.1017/S002211209800860X

[48] Uzunoğlu, B., M. Tan, and W. G. Price. "Low-Reynolds-number flow around an oscillating circular cylinder using a cell viscousboundary element method." International Journal for Numerical Methods in Engineering 50, no. 10 (2001): 2317-2338. https://doi.org/10.1002/nme.122 